\title{
STRASBOURG-ESO CATALOGUE OF GALACTIC PLANETARY NEBULAE
}

\author{
ACKER AGNĖS and OCHSENBEIN FRANÇOIS \\ Observatoire de Strasbourg, 11, rue de l'Université 67000 STRASBOURG, France
}

\begin{abstract}
A list of 1820 objects, each of them called at least once a planetary nebula, has been inspected; 1143 of them have been classified as true or probable planetary nebulae by the authors of the catalogue (Tables 1$) ; 347$ objects, the status of which is still unclear, were classified among the "possible" planetary nebulae (Table 2).; 330 objects have been rejected, on various grounds, from the community of planetary nebulae (Table 3).

The Catalogue consists of two parts. Part I includes the following chapters:

A Explanations of the Catalogue

B Tables

C References of papers containing 20 objects or more

D Finding Charts

A new designation system for planetary nebulae following the recommendations of IAU Commission 5 (Astronomical Nomenclature) was introduced here with the structure PN G $\ell \ell \ell . \ell \pm b b . b$ where $\mathbf{P N}$ means 'Planetary Nebula', G means 'Galactic coordinates' and $\ell \ell \ell . \ell \pm b b . b$ stands for the galactic longitude and latitude respectively, truncated to one decimal place only. Among the 1143 true PN, 846 come from the CGPN, 218 from the supplementary lists compiled by Kohoutek, and 79 were discovered recently. Of the 347 possible PN, 21 come from the CGPN and 82 from the supplementary lists. Of the 330 misclassified PN, 169 are from the CGPN and 101 from the supplementary lists.

Finding charts have been constructed for all true planetary nebulae in the catalogue. The identification was possible either from the finding charts provided by the discoverer, appearing mainly in the CGPN, or from information communicated by the observers in the framework of the spectroscopic survey.

In the main catalogue (Part II), the following data are given for each of the 1143 PNs: PN designation and names, discovery lists (cross-identifications), coordinates (1950 and 2000), diameters (optical and radio), radial and expansion velocities, relative line intensities, fluxes in $H \beta$, IR and radio, central star parameters, distances and notes, and bibliography (1065-1991).

Histograms illustrate the distributions of the galactic positions, the angular diameters, the radial (577) and expansion (284) velocities, and the fluxes in $H \beta$ (991), at $6 \mathrm{~cm}(655)$, and in IR from IRAS (788).
\end{abstract}

